Urol. Prax. 2020 -22:93-99

https://doi.org/10.1007/s41973-020-00103-0

Online publiziert: 5 . August 2020

(c) Der/die Autor(en) 2020
Florian Schmid • Michaela Mack - Tullio Sulser

Klinik für Urologie, UniversitätsSpital Zürich, Zürich, Schweiz

\section{Die Harnröhrenstriktur des Mannes}

\section{Teil 1: Ätiologie, Diagnostik und minimal- invasive Therapie}

\section{Einleitung}

Die Urethrastriktur des Mannes stellt eine Herausforderung im Alltag des Urologen dar und geht mit einer veritablen Einschränkung der Lebensqualität betroffener Patienten einher [46]. Harnröhrenstrikturen verursachen durch eine funktionelle Obstruktion eine kontinuierliche Schädigung des gesamten Harntrakts, und die Erkrankung kann bei chronischem Verlauf bis zur Einschränkung der Nierenfunktion fortschreiten [48]. Die Entität wird oftmals stiefmütterlich behandelt - der Fakt, dass es von der europäischen Gesellschaft für Urologie (EAU) keine Guidelines zu dieser Thematik gibt, verleiht diesem Umstand Gewicht. Zuletzt wurden internationale Richtlinien vom amerikanischen Pendant - der American Urology Association (AUA) - im Jahr 2017 publiziert [47]. Die Prävalenz in der Gesamtbevölkerung liegt bei knapp $1 \%$, wobei circa die Hälfte aller Fälle entweder einen iatrogenen oder idiopathischen Ursprung haben [24]. Die Inzidenz der Harnröhrenstrikturen nimmt mit zunehmendem Alter exponentiell $\mathrm{zu}$ und beginnt bei Patienten mit über 65 Jahren deutlich anzusteigen auf über 100 Fälle pro 100.000 Einwohner [33]. Anatomisch wird die Harnröhre in einen anterioren und posterioren Anteil eingeteilt [24]. Die prostatische und membranöse Harnröhre zählen zum posterioren Abschnitt. Engstellen der bulbären, penilen und glandulären Harnröhre sowie des Meatus urethrae externus werden als anteriore Harnröhrenstrikturen be- zeichnet und sind weitaus häufiger. Nach entsprechender Diagnostik lässt sich die Einteilung in kurzstreckige $(\leq 1 \mathrm{~cm})$ oder langstreckige $(>1 \mathrm{~cm})$ Strikturen vornehmen. Die bulbäre Harnröhre ist unter den anterioren Harnröhrenstrikturen am häufigsten betroffen (ca. 50\% aller Fälle) und hat prinzipiell die günstigste Prognose vorzuweisen ([13]; - Abb. 1). Der Grund hierfür liegt in der guten Durchblutung des Gewebes durch das kräftig ausgebildete und umliegende Corpus spongiosum [35].

Das potenzielle therapeutische Spektrum in der Behandlung einer Urethrastriktur ist, ungeachtet der Lokalisation, breit: Bougierung, Dilatation, innere Urethrotomie (Harnröhrenschlitzung) oder eine offene Rekonstruktion stehen zur Auswahl [1]. Insbesondere Patienten mit einem Strikturrezidiv sind jedoch schwierig zu behandeln. Die richtige therapeutische Massnahme muss stets individuell und in Absprache mit dem Patienten getroffen werden. Eine vorangehende, detaillierte Diagnostik ist aber entscheidend, um den richtigen Therapiepfad einzuschlagen. Auch in der Diagnostik steht uns ein mannigfaltiges Angebot an Untersuchungen zur Verfügung: Vom Uroflow und der sonographischen Restharnmessung über die retrograde Urethrographie, die Miktionzysturethrographie und die Zystoskopie bis hin zur eher selten indizierten Urodynamik. Besonders hohe Anforderungen sind an die Behandlung von Urethrastrikturen zu stellen, welche durch innate Anomalien bedingt sind. So ist $\mathrm{z}$. B. die Behebung einer Engstelle nach operativer Korrektur einer
Hypospadie von ausgeprägter Komplexität begleitet [31]. Die Harnröhrenstriktur bei der Frau ist äusserst selten und wird im Rahmen dieses Reviews keine Thematisierung erfahren.

In diesem ersten Teil thematisieren wir die Ätiologie und Pathophysiologie, Symptomatik, Diagnostik und die minimalinvasive Therapie bei den unterschiedlichen Formen von Harnröhrenstrikturen. Im einem späteren, zweiten Teil fokussieren wir uns auf die offene, operative Rekonstruktion der Harnröhre.

\section{Ätiologie und Pathophysiologie}

Die Entstehung einer Urethrastriktur ist in den meisten Fällen entweder idiopathisch oder iatrogen [28]. Iatrogene Ursachen sind häufig nach Interventionen oder Manipulationen an der Harnröhre zu finden (z. B. nach traumatischer Dauerkathetereinlage, Zystoskopie, transurethraler Resektion, operativer Hypospadiekorrektur oder radikaler Prostatektomie). Nach transurethralen Eingriffen (z. B. TUR-Blase oder TUR-Prostata) ist die Harnröhrenstriktur eine der häufigsten Spätkomplikationen. Weitere Gründe können Beckentraumata mit Begleitverletzung der Urethra [16] oder infektiöse Urethritiden sein [33]. Die Sequelae Letzterer sind in der westlichen Welt, aufgrund rechtzeitiger Diagnose und adäquater antibiotischer Therapie, mittlerweile selten geworden. Sie können theoretisch jedoch zu einer Inflammation der Harnröhre führen und nach Abheilung eine Striktur hinterlassen [17]. 

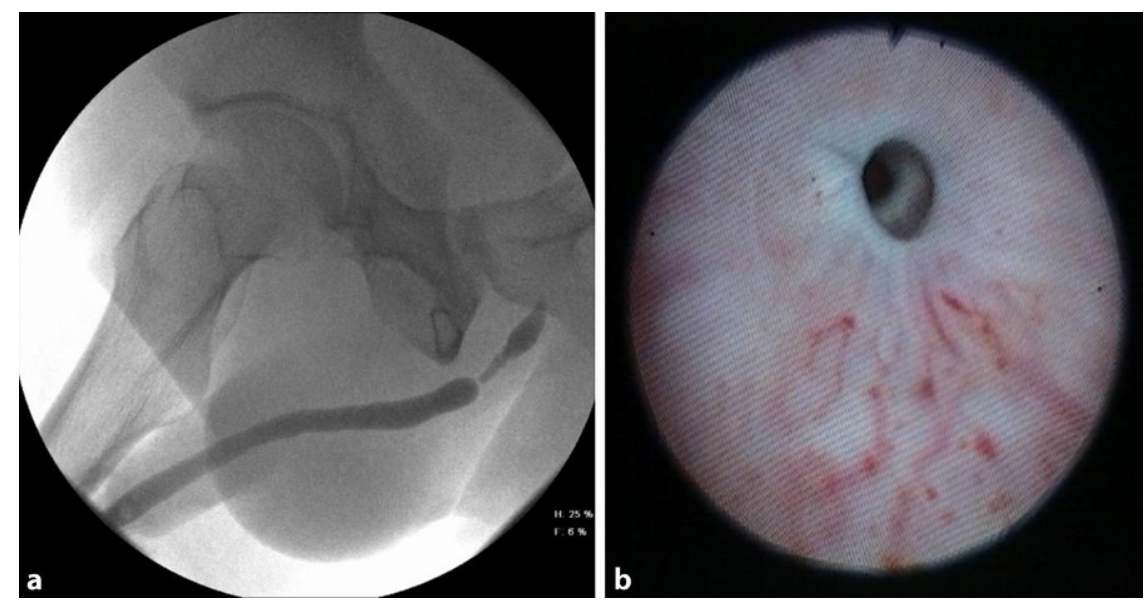

Abb. $1 \Delta$ Darstellung einer bulbären Urethrastriktur mittels retrograder Urethrographie (a).Zystoskopischer Befund der bulbären Urethrastriktur (b)

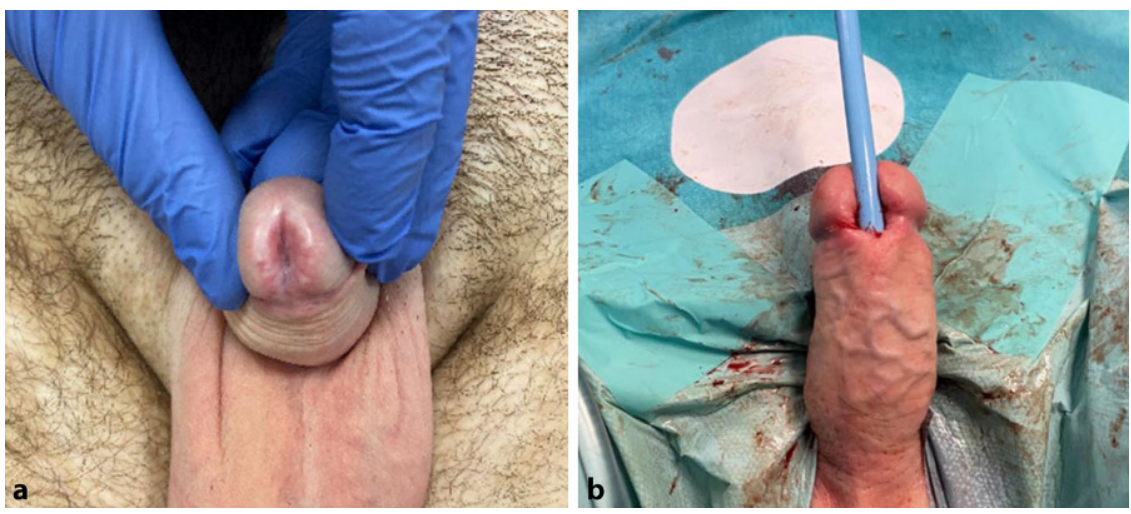

Abb. $2 \Delta$ Meatusstenose mit klinischem Befund eines Lichen sclerosus et atrophicans (a). Intraoperativer Befund einer offenen Meatusplastik (b)

Histologisch findet sich ein narbiger Umbau des subepithelialen Gewebes (urethranah) bis hin zum Corpus spongiosum vor, wobei es konsekutiv $\mathrm{zu}$ einer Verengung des Lumens kommt [35]. Die pathophysiologische Abfolge kann in folgende Schritte unterteilt werden: Ein schädlicher Stimulus (mechanisch, chemisch oder infektiös) geht einer Plattenepithelmetaplasie voraus, wobei dadurch Fissuren im Epithelium entstehen. Es kommt dann $\mathrm{zu}$ einer Extravasation von initial Entzündungszellen und anschliessend Fibroblasten aus der Blutbahn mit konsekutiver Fibrose des subepithelialen Gewebes. Die dabei entstehenden Inseln an fibrotischen Plaques können sich im weiteren Verlauf verbinden und sich bis ins Corpus spongiosum ausbreiten. Wenn es dabei zu einer zirkumferenten Vereini-
Harnstrahl, verlängerte Miktionszeit, Nachträufeln, Restharngefühl und erhöhte Miktionsfrequenz (Pollakisurie) sind nicht selten auch mit verstärktem Harndrang oder Dysurie assoziiert. Die klinische Präsentation bei Erstmanifestation ist auch bereits mit Harnverhalt oder gar chronischer Retentionsblase mit oder ohne Schädigung des oberen Harntrakts, Makrohämaturie oder rezidivierenden Harnweginfektionen möglich. Beim Vorliegen einer distalen Harnröhrenstriktur berichten Patienten mitunter von einer teils ausgeprägten Palmurie (divergierender Harnstrahl), welche einen störenden Einfluss auf die Harnblasenentleerung im öffentlichen oder heimischen Umfeld haben kann.

\section{Diagnostik}

Zur Diagnostik zählt nach erfolgter Anamnese als Erstes die körperliche Untersuchung. Obwohl ein Grossteil der Urethra der klinischen Untersuchung nicht zugänglich ist, kann man mit der Inspektion von Meatus, Glans und Praeputium das Vorliegen einer Meatusstenose oder eines Lichen sclerosus ausschliessen [14]. Mit der Ultraschalluntersuchung verifizieren wir den vorliegenden Restharn in der Blase post mictionem, können die Harnblasenwanddicke abschätzen und eine Aussage über den oberen Harntrakt (Nieren und proximaler Harnleiter) treffen. Der vorangehende Uroflow lässt als einfache und kostengünstige Untersuchung den Verlauf der Harnblasenentleerung objektivieren [8]. Dafür wird mittels Miktionsmenge (ml) über die Zeit (s) eine Kurve abgeleitet, wobei eine Striktur sich durch eine plateauförmig abgeflachte und verlängerte Miktionskurve auszeichnet (-Abb. 3). Diese Methode eignet sich einerseits zur objektiven Unterscheidung von einer normalen Miktion und wird andererseits häufig auch nach der Behandlung zur Kontrolle des Therapieerfolgs oder der frühzeitigen Erkennung eines Rezidivs verwendet [43]. Aus neurourologischer Sicht kann jedoch eine, entweder durch gutartige Prostatahyperplasie oder Urethrastriktur bedingte, infravesikale Obstruktion nicht durch einen Uroflow, sondern lediglich mittels Urodynamik diagnostiziert werden. $\mathrm{Zu}$ - 
dem ist auch eine Aussage über die Länge der Striktur anhand des Uroflows nicht möglich. Der Goldstandard für die Diagnostik der Harnröhrenstriktur ist die retrograde Urethrographie [32]. Die Lage und Länge der Striktur lässt sich damit gut darstellen und anatomisch lokalisieren. Obwohl die Untersuchung in der Literatur mit einer hohen Sensitivität (75-100\%) und Spezifität (72-92\%) angegeben wird [29], ist sie stark untersucherabhängig, und die Interpretation der Befunde kann sich vom intraoperativen Befund teilweise erheblich unterscheiden [2]. Daher muss auf eine möglichst orthograde Röntgeneinstrahlung und ein geradlinig ausgerichtetes Glied geachtet werden. Um die proximale (posteriore) Harnröhre ebenfalls gut mitbeurteilen zu können, kann zusätzlich eine antegrade Urethrographie resp. eine Miktionszysturethrographie (MCUG) durchgeführt werden. Hier können der Blasenhals (z.B. mit Frage nach Sklerosierung) und die Eröffnung der prostatischen Harnröhre während der Miktion eingesehen und beurteilt werden [35]. Falls die Einmalkatheterisierung zur retrograden Füllung der Harnblase mit Kontrastmittel aufgrund der ausgeprägten oder absoluten Urethrastriktur nicht gelingt und/oder gleichzeitig sogar ein Harnverhalt vorliegt, muss eine Dauerkathetereinlage via suprapubische Zystostomie veranlasst werden. Die Urethrozystoskopie komplettiert die standardmässige Diagnostik bei Patienten mit Harnröhrenstriktur. Sie wird oftmals bei unklarer Bildgebung als Ergänzung eingesetzt und hat die Einschränkung, dass hinter einer nichtpassierbaren Striktur liegende Veränderungen nicht eingesehen werden können. So können die genaue Länge der Striktur oder weitere, dahinterliegende Engstellen nicht oder nur limitiert beurteilt werden. Im Falle einer bereits angelegten, suprapubischen Zystostomie kann die Urethrozystoskopie von ,antegrad“ komplettiert werden [18]. Die Zystoskopie kann, sofern präoperativ die Striktur überhaupt passierbar ist, einem Operateur zur Vorbereitung und Planung des transurethralen oder offenen operativen Eingriffs ergänzende Einblicke liefern [19]. Andernfalls muss die Beurteilung der gesamten Harn-

Urol. Prax. 2020 ·22:93-99 https://doi.org/10.1007/s41973-020-00103-0

(c) Der/die Autor(en) 2020

\section{F. Schmid $\cdot$ M. Mack $\cdot$ T. Sulser}

\section{Die Harnröhrenstriktur des Mannes. Teil 1: Ätiologie, Diagnostik und minimal-invasive Therapie}

\section{Zusammenfassung}

Die Harnröhrenstriktur des Mannes ist eine komplexe Erkrankung und geht mit einer signifikant eingeschränkten Lebensqualität sowie oftmals wiederkehrenden Behandlungen durch den Urologen einher. Abhängig vom Schweregrad und der Dauer seit der ersten Symptommanifestation kann sich das Krankheitsbild u. U. auch negativ auf den gesamten Harntrakt auswirken. Je nach anatomischer Lokalisation der Urethraengstelle stehen zudem unterschiedliche Therapieoptionen zur Wahl. Daher ist es entscheidend, dass der Wahl des Therapiepfads eine saubere Diagnostik und eine ganzheitliche Aufklärung mit gemeinsamer Entscheidungsfindung zwischen Patient und Arzt vorausgeht. Nur so kann, unter Berücksichtigung der individuellen Präferenzen, die richtige Behandlung gewählt und ein befriedigendes Resultat herbeigeführt werden. In diesem zweiteiligen Review wird die Urethrastriktur des Mannes von der Ätiologie über die Diagnostik bis hin zur operativen Behandlung ausgeleuchtet.

\section{Schlüsselwörter}

Harnröhrenverengung · Urethraobstruktion · Harnröhrenstenose · Urethrastriktur . Abgeschwächter Harnstrahl

\section{La sténose urétrale chez l'homme. 1re partie: étiologie, diagnostic, traitement mini-invasif}

\section{Résumé}

La sténose urétrale chez l'homme est une maladie complexe causant une perturbation significative de la qualité de vie et exigeant souvent des traitements répétés chez l'urologue. Selon sa sévérité et le temps écoulé depuis la première manifestation de symptômes, cette maladie peut éventuellement avoir un impact négatif sur tout l'ensemble de l'appareil urinaire. On dispose de différentes options thérapeutiques en fonction de la localisation anatomique de la sténose urétrale. II est donc essentiel pour le choix du parcours thérapeutique d'établir un diagnostic solide et une éducation holistique avec une prise de décisionen compte conjoin- te entre le patient et le médecin de tous les aspects du patient et de prendre les décisions en commun avec le patient. Ce n'est qu'ainsi, avec égard aux préférences individuelles, qu'on pourra choisir le traitement approprié et atteindre un résultat satisfaisant. Cette revue en deux parties examine la sténose urétrale de l'homme et informe sur l'étiologie, le processus de diagnostic et le traitement chirurgical.

\section{Mots clés}

Constriction urétrale . Obstruction urétrale . Sténose urétrale $\cdot$ Stricture urétrale $\cdot$ Faible jet d'urine röhre und das Einsehen der Harnblase intraoperativ nachgeholt werden. Dabei achtet man sich auf das Vorhandensein von Harnblasendivertikel als Hinweise für eine beginnende Dekompensation oder ein simultanes Vorliegen einer (z.B. malignen) Blasenpathologie. Der Stellenwert der Urodynamik vor operativer Korrektur wird kontrovers diskutiert und selten eingesetzt. Trotzdem sollte eine begleitende Harnblasenfunktionsstörung (z. B. Entleerungsstörung aufgrund atoner oder hypoaktiver Harnblase) bei leisestem Verdacht vor operativer Sanierung mitunter abgeklärt werden.

\section{Minimal-invasive Therapie}

Die Möglichkeiten der operativen Therapie sind in entweder offene oder endourologische Verfahren einzuteilen. Bei den endourologischen Interventionen stehen uns entweder die Harnröhrenbougierung oder die transurethrale Urethrotomia interna nach Sachse zu Verfügung. Die Möglichkeiten zur offenen Rekonstruktion einer Harnröhrenstriktur mittels unterschiedlicher und operativ teils anspruchsvollen Plastiken (End-zu-End, Flap-Technik oder Transplantation von autologem Gewebe) ist vielseitig und 


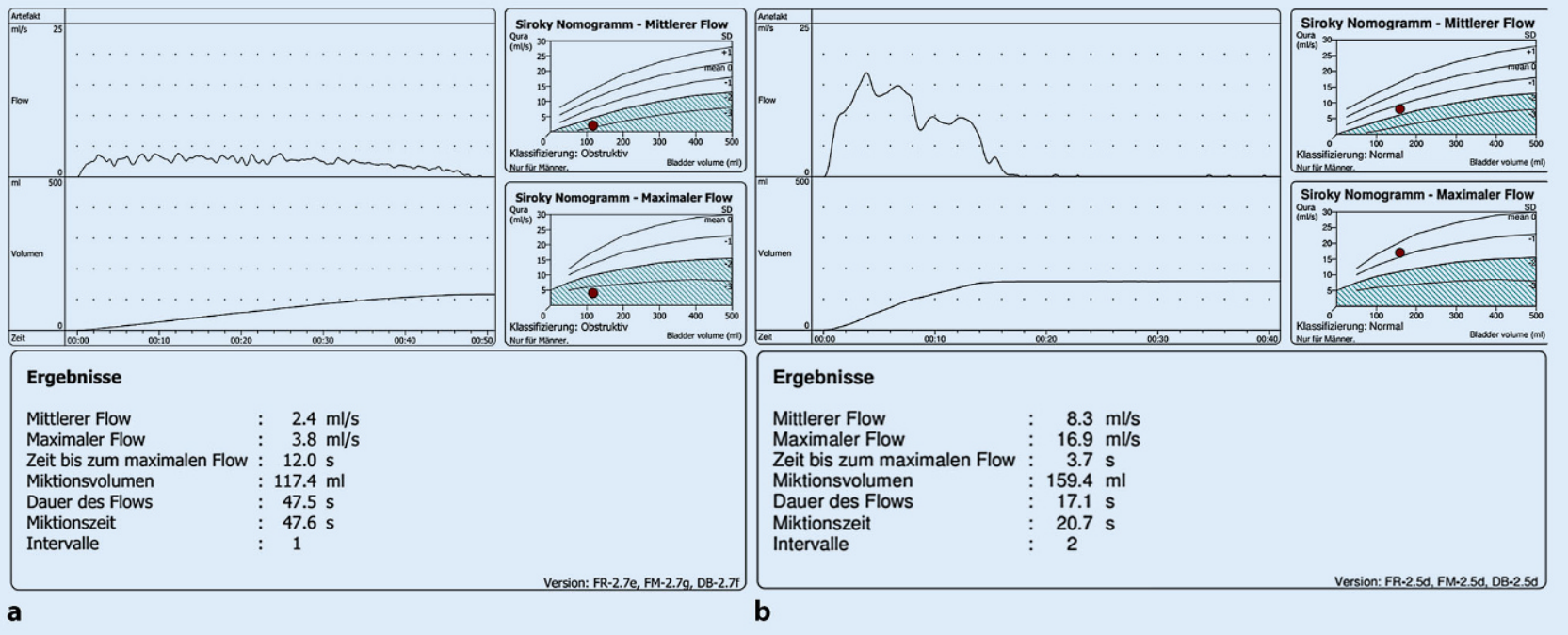

Abb. $3 \Delta$ Uroflow mit plateauförmig abgeflachter und zeitlich verlängerter Miktion (a). Nach erfolgreicher Bougierung (b)

wird im Rahmen des nächsten Artikels im Detail ausgeleuchtet werden. Das Vorliegen einer distalen Harnröhrenstrikur (z.B. Meatusstenose) und die verschiedenen Formen der Meatotomie sind ebenfalls zu berücksichtigen.

\section{Bougierung und Dilatation}

Kurze und passierbare Strikturen können probatorisch mittels Bougierungen behandelt werden. Für die Dilatation werden Einmalkatheter oder dafür spezifisch geeignete Bougierstifte verwendet, wobei mit einem tiefen Durchmesser (Charrière 10) begonnen und anschliessend sukzessive bis zum einem höhergradigen (Charrière 22 oder 24) gesteigert wird. Das Gewebe wird durch die Dehnung nur minimal traumatisiert, und der unmittelbare Effekt ist für den Patienten ausgezeichnet (•Abb. 3). Die Vorteile einer ambulanten, gut erträglichen Behandlung ohne Notwendigkeit einer Narkose mit kaum vergesellschafteten Schmerzen liegen auf der Hand. Leider liegt jedoch die Rezidivrate innerhalb von 2 Jahren postoperativ mit bis zu $85 \%$ aller behandelten Fälle äusserst hoch [44]. Natürlich können die Dilatationen wiederholt und in regelmässigen Zeitintervallen durchgeführt werden - es zeigt sich bei diesen Patienten jedoch, auch im Falle des Erlernens eines Selbstkatheterismus mit weichen Bougier-Instrumenten, eine deut- lich eingeschränkte Lebensqualität [27]. Nach anfänglichem Enthusiasmus wurde von der Einlage permanenter Platzhalter im Sinne von intraurethralen Stents (z. B. UroLume ${ }^{\circledR}$, American Medical Systems Inc., MN, USA oder Memokath ${ }^{\circledR}$, Pnn Medical A/S, Kvistgaard, Denmark) zunehmend abgesehen. Die Langzeitresultate sind, nicht zuletzt wegen teils ausgeprägten Fremdkörperreaktionen, äusserst schlecht und die Komplikationsraten mit schlussendlicher Notwendigkeit von komplexen Salvage-Operationen hoch [20].

\section{Urethrotomie}

Die innere Sichturethrotomie nach Sachse ist eine valable Alternative zur Bougierung und kann mittels relativ simplem, transurethralem Eingriff in einem ambulanten oder kurzstationären Aufenthalt vorgenommen werden. Hierbei wird eine Inzision bei 12 Uhr durchgeführt und anschliessend zur Abheilung ein Platzhalter (transurethraler Dauerkatheter) für einige Tage eingelegt (je nach Präferenz des Operateurs und Länge der Striktur zwischen 2-7 Tagen; - Abb. 4). Insbesondere im Bereich der membranösen Harnröhre muss die Operation jedoch mit grösster Vorsicht ausgeführt werden, damit der darunterliegende Schliessmuskel (Sphincter urethrae externus) unter keinen Umständen verletzt wird. Bei
Ausbleiben eines Therapieerfolgs mit frühzeitigem Rezidiv, wobei auch bei diesem Verfahren mit einer Misserfolgsrate in $40-85 \%$ der Behandlungen innerhalb von bis zu 4 Jahren zu rechnen ist, sollte konsekutiv eine offene Rekonstruktion favorisiert werden [37]. Die besten Resultate sind nach erstmaliger Urethrotomie bei primären, kurzstreckigen Strikturen $(<1 \mathrm{~cm})$ im bulbären Bereich der Harnröhre zu erwarten mit einer Rezidivrate von „lediglich“ ca. 30\% [30]. Die anhaltenden Erfolgschancen bei repetitiven Sichturethrotomien sind deutlich geringer mit bei einem zweiten Versuch lediglich $4 \%$ und einem dritten Versuch gegen $0 \%$ [37]. In erfahrenen Händen spielt es keine Rolle, ob zuerst der therapeutische Versuch einer SachseUrethrotomie oder gleich der einer primären, offenen Harnröhrenrekonstruktion gewählt wird [3]. Je nach Literatur und Zentrum wird nach mehrmaligen Harnröhrenschlitzungen aber von einer höheren Misserfolgsquote nach sekundärer Harnröhrenplastik berichtet [45]. Zusammenfassend lässt sich sagen, dass gemäss den international publizierten Daten eine Harnröhrenschlitzung nach Sachse nur bei Patienten mit bulbären und kurzstreckigen Strikturen zu empfehlen ist - denn lediglich da besteht die Aussicht auf einen anhaltenden Erfolg mit minimalem Aufwand. 

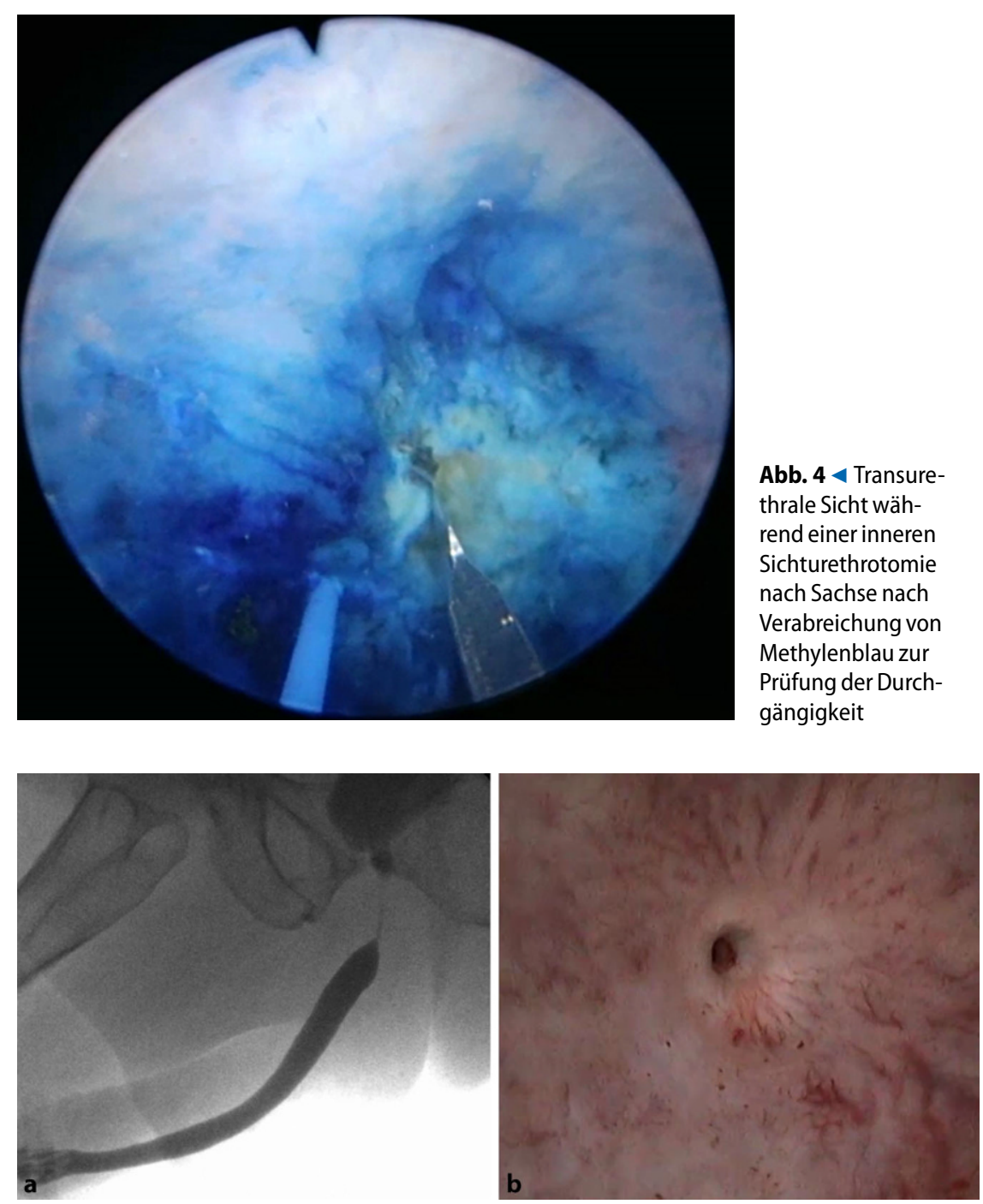

Abb. $5 \Delta$ Retrograde Urethrographie (a) und zystoskopischer Befund einer langstreckigen vesikourethralen Anastomosenstriktur (b) bei einem 73-jährigen Patienten mit St. n. radikaler Prostatektomie und Salvage-Radiatio

\section{Meatotomie}

Ganz distale Harnröhrenstrikturen umfassen Meatusstenosen, Engstellen der Fossa navicularis und glanduläre Harnröhrenstrikturen. Mit bis zu einem Fünftel der Fälle repräsentieren diese Strikturen einen relevanten Anteil der anterioren Harnröhrenstrikturen. Sie sind einer operativen Versorgung generell gut zugänglich. Eine Meatusstenose kann entweder mittels offener Meatusplastik oder aber durch eine einfache, erweiterte oder plastische Meatotomie versorgt werden. Bei längerstreckigen Strikturen mit Beteiligung der penilen Harnröhre oder vorangehenden Hypospadieoperationen stellt die offene Rekonstruktion oftmals
Abb. $4<$ Transurethrale Sicht während einer inneren Sichturethrotomie nach Sachse nach Verabreichung von Methylenblau zur Prüfung der Durchgängigkeit

zusätzlich mit resorbierbarem Faden (z.B. Vicryl rapid) quer zur Inzisionrichtung vernäht werden (•Abb. 2). Die Nachsorge ist simpel: Die postoperative Katheterverweildauer von 2-7 Tagen kann grosso modo mit der Sachse-Urethrotomie verglichen werden, wobei bei vorliegendem Lichen sclerosus je nach Präferenz zur lokalen Nachbehandlung zusätzlich noch topische Kortikosteroide eingesetzt werden können [9]. Der Eingriff zeichnet sich grundsätzlich durch eine hohe Patientenzufriedenheit aus und hat den Vorteil, dass er im weiteren Verlauf stufenweise, sofern auch wirklich nötig, mit einer offenen Meatusplastik ergänzt werden kann [34]. Die Erfolgsrate liegt deutlich höher im Vergleich $\mathrm{zu}$ anderen Formen der endoskopischen Harnröhrenstrikturbehandlung, und es kann mit einer langfristigen Heilungsrate von bis $\mathrm{zu} 85 \%$ gerechnet werden [34].

Zum Schluss soll noch auf die Wichtigkeit eines zufriedenstellenden kosmetischen Ergebnisses hingewiesen sein. Der Goldstandard der Behandlung bei längerstreckigen Strikturen mit zusätzlichem Betreff der Fossa navicularis, glandulären und penilen Harnröhre ist die offene Rekonstruktion mit Verwendung eines autologen Transplantats (z. B. Mundschleimhaut) oder einer gestielten Lappenplastik in möglichst einzeitigem Verfahren [23]. Oftmals werden jedoch zweizeitige Eingriffe mit initialer Resektion des Narbengewebes, Defektdeckung durch das autologe Transplantat, Abwarten einer Abheilung der neuen Urethraplatte und nach 3-6 Monaten schliesslich die sekundäre Tubularisierung mit definitivem Hautverschluss notwendig [39]. Diese Art von Eingriffen werden im Rahmen des nächsten Artikels ebenfalls beschrieben und ausführlich diskutiert werden.

\section{Vesikourethrale Anastomosen- striktur}

iür zuerst das Urethrotom eingefüh und auf den gewünschten Durchmesser dilatiert wird. Anschliessend wird durch das Urethrotom eine Klinge gezogen und somit die Harnröhre dorsal oder ventral länglich eingeschnitten [21]. Um ein erneutes Verkleben der Schnittflächen zu verhindern, kann die Inzision
Separat zu betrachten sind die Strikturen der vesikourethralen Anastomose nach radikaler Prostatovesikulektomie zur Behandlung von Patienten mit lokalisiertem Prostatakarzinom [11]. Obwohl sie zu den eher seltenen Komplikationen gehö- 
ren, sind sie als schwerwiegend zu werten und deren Behandlung oftmals langwierig und mühsam [15]. Dazu kommt, dass die Evidenz schwach ist und sich lediglich auf retrospektive Analysen mit begrenzten Fallzahlen oder auf Expertenmeinungen abstützt [38]. Demzufolge sind die Diagnostik und Abfolge der Therapie wenig standardisiert und stellen den behandelnden Urologen vor eine echte Herausforderung [6]. Die Patienten sind durch erhebliche funktionelle Einschränkungen mit einer deutlichen Verschlechterung der Lebensqualität im Alltag konfrontiert [46]. In früheren Jahren lag die Rate der Anastomosenstrikturen bei bis zu $15 \%$ [42]. Sie konnten durch Verbesserung der Operationstechnik und durch die Etablierung minimalinvasiver Verfahren aufheute 5-8 \% reduziert werden [22]. Die Latenzzeit bis zum ersten Auftreten dauert normalerweise etwa 4-6 Monate nach radikaler Prostatektomie. Deutlich schlechtere Resultate und höhere Strikturraten der Anastomosen sind nach Salvage-Prostatektomie bei vorangehender Strahlentherapie mit initial kurativer Absicht zu erwarten [41]. Zudem stellt die adjuvante Strahlentherapie respektive auch die frühe SalvageRadiatio ein erhöhtes Risiko für eine Fibrosierung des Gewebes mit konsekutiver Strikturausbildung dar [7]. Wichtige Komorbiditäten, welche gemäss Literatur ein erhöhtes Risiko einer vesikourethralen Anastomosenstriktur nach Prostatektomie mit sich bringen, sind ein Diabetes mellitus, arterielle Hypertonie, Mikroangiopathie und eine positive Raucheranamnese ([5]; - Abb. 5). Das therapeutische Spektrum bewegt sich zwischen Bougierung, Blasenhalsinzision, Urethrotomia nach Sachse, transurethraler Resektion und offener Rekonstruktion des Blasenhalses [47]. Die Bougierung verfügt über eine erstaunlich hohe Erfolgsrate von bis zu $85 \%$ nach einer einmaligen Behandlung [4]. Deutlich schlechtere Zahlen sind mit der Blasenhalsinzision nach Turner-Warwick vergesellschaftet (Rezidivrate bis zu $60 \%$ ) [37]. Regelmässige Selbstbougierung oder der Einsatz von Dilatatoren (z.B. Memokath ${ }^{\circledR}$ ) können im Falle von Rezidiven optional eingesetzt werden [36]. Eine alleinige SachseUrethrotomie ist langfristig meist nicht ausreichend und oftmals eine simultane Erweiterung im Sinne einer Blasenhalsinzision oder alternativ die Eröffnung der Striktur durch Verwendung von Laser oder der transurethralen Resektion notwendig $[6,12]$. Falls die endourologischen Verfahren wiederholt keinen anhaltenden Erfolg zeigen ( $\geq 2$ Rezidive), muss mit dem Patienten über eine offene, perineale Reanastomosierung (Endzu-End) diskutiert werden [40]. Diese Therapieoption kommt im Rahmen des zweiten Teils ebenfalls zur Sprache.

\section{Korrespondenzadresse}

\begin{tabular}{l}
$\begin{array}{l}\text { Dr. med. Florian Schmid } \\
\text { Klinik für Urologie, } \\
\text { UniversitätsSpital Zürich } \\
\text { Frauenklinikstrasse 10, } \\
8091 \text { Zürich, Schweiz } \\
\text { Florian.Schmid@usz.ch }\end{array}$ \\
$\begin{array}{l}\text { Dr. med. Michaela Mack } \\
\text { Klinik für Urologie, } \\
\text { UniversitätsSpital Zürich } \\
\text { Frauenklinikstrasse 10, } \\
8091 \text { Zürich, Schweiz } \\
\text { Michaela.Mack@usz.ch }\end{array}$ \\
$\begin{array}{l}\text { Prof. Dr. med. Tullio Sulser } \\
\text { Klinik für Urologie, } \\
\text { UniversitätsSpital Zürich } \\
\text { Frauenklinikstrasse 10, } \\
\text { 8091 Zürich, Schweiz } \\
\text { Tullio.Sulser@usz.ch }\end{array}$ \\
\hline
\end{tabular}

Funding. Open access funding provided by University of Zurich

\section{Einhaltung ethischer Richtlinien}

Interessenkonflikt. F. Schmid, M. Mack und T. Sulser geben an, dass kein Interessenkonflikt besteht.

Für diesen Beitrag wurden von den Autoren keine Studien an Menschen oder Tieren durchgeführt. Für die aufgeführten Studien gelten die jeweils dort angegebenen ethischen Richtlinien.

Open Access. Dieser Artikel wird unter der Creative Commons Namensnennung 4.0 International Lizenz veröffentlicht, welche die Nutzung, Vervielfältigung, Bearbeitung, Verbreitung und Wiedergabe in jeglichem Medium und Format erlaubt, sofern Sie den/die ursprünglichen Autor(en) und die Quelle ordnungsgemäß nennen, einen Link zur Creative Commons Lizenz beifügen und angeben, ob Änderungen vorgenommen wurden.

Die in diesem Artikel enthaltenen Bilder und sonstiges Drittmaterial unterliegen ebenfalls der genannten Creative Commons Lizenz, sofern sich aus der Abbil- dungslegende nichts anderes ergibt. Sofern das betreffende Material nicht unter der genannten Creative Commons Lizenz steht und die betreffende Handlung nicht nach gesetzlichen Vorschriften erlaubt ist, ist für die oben aufgeführten Weiterverwendungen des Materials die Einwilligung des jeweiligen Rechteinhabers einzuholen.

Weitere Details zur Lizenz entnehmen Sie bitte der Lizenzinformation auf http://creativecommons.org/ licenses/by/4.0/deed.de.

\section{Literatur}

1. Anger JT, Buckley JC, Santucci RA, Elliott SP, Saigal CS, Urologic Diseases in America Project (2011) Trends in stricture management among male medicare beneficiaries: underuse of urethroplasty? Urology 77(2):481-485. https://doi.org/10. 1016/j.urology.2010.05.055

2. Bach P, Rourke K (2014) Independently interpreted retrograde urethrography does not accurately diagnose and stage anterior urethral stricture: the importance of urologist-performed urethrography. Urology 83(5):1190-1193. https://doi.org/10. 1016/j.urology.2013.12.063

3. Barbagli G, Palminteri E, Lazzeri M, Guazzoni G, Turini D (2001) Long-term outcome of urethroplasty after failed urethrotomy versus primary repair. J Urol 165(6 Pt 1):1918-1919. https://doi.org/10. 1097/00005392-200106000-00018

4. Besarani D, Amoroso P, Kirby R (2004) Bladder neck contracture after radical retropubic prostatectomy. BJU Int 94(9):1245-1247. https://doi.org/10.1111/ j.1464-410X.2004.05151.x

5. Borboroglu PG, Sands JP, Roberts JL, Amling CL (2000) Risk factors for vesicourethral anastomotic stricture after radical prostatectomy. Urology 56(1):96-100. https://doi.org/10.1016/s00904295(00)00556-2

6. Browne BM, Vanni AJ (2017) Management of urethral stricture and bladder neck contracture following primary and salvage treatment of prostate cancer. Curr Urol Rep 18(10):76. https:// doi.org/10.1007/s11934-017-0729-0

7. Chade DC, Eastham J, Graefen M, Hu JC, Karnes RJ, Klotz L, Montorsi F, van Poppel H, Scardino PT, Shariat SF (2012) Cancer control and functional outcomes of salvage radical prostatectomy for radiation-recurrent prostate cancer: a systematic review of the literature. Eur Urol 61(5):961-971. https://doi.org/10.1016/j.eururo.2012.01.022

8. Chan CK, Yip SK, Wu IP, Li ML, Chan NH (2012) Evaluation of the clinical value of a simple flowmeter in the management of male lower urinary tract symptoms. BJU Int 109(11):1690-1696. https:// doi.org/10.1111/j.1464-410X.2011.10581.x

9. Chi CC, Kirtschig G, Baldo M, Brackenbury F, Lewis F, Wojnarowska $F$ (2011) Topical interventions for genital lichen sclerosus. Cochrane Database Syst Rev 12:CD8240. https://doi.org/10.1002/ 14651858.CD008240.pub2

10. Dielubanza EJ, Han JS, Gonzalez CM (2014) Distal urethroplasty for fossa navicularis and meatal strictures. Transl Androl Urol 3(2):163-169. https:// doi.org/10.3978/j.issn.2223-4683.2014.04.02

11. Elliott SP, Meng MV, Elkin EP, McAninch JW, Duchane J, Carroll PR, Ca PI (2007) Incidence of urethral stricture after primary treatment for prostate cancer: data from CaPSURE. J Urol 178(2):529-534. https://doi.org/10.1016/j.juro. 2007.03.126 (discussion 534) 
12. Eltahawy E, Gur U, Virasoro R, Schlossberg SM, Jordan GH (2008) Management of recurrent anastomotic stenosis following radical prostatectomy using holmium laser and steroid injection. BJU Int 102(7):796-798. https://doi.org/10.1111/j.1464410X.2008.07919.x

13. Fenton AS, Morey AF, Aviles R, Garcia $C R$ (2005) Anterior urethral strictures: etiology and characteristics. Urology 65(6):1055-1058. https:// doi.org/10.1016/j.urology.2004.12.018

14. Garaffa G, Shabbir M, Christopher N, Minhas S, RalphDJ(2011) The surgical management of lichen sclerosus of the glans penis: our experience and review of the literature. JSex Med 8(4):1246-1253. https://doi.org/10.1111/j.1743-6109.2010.02165.

15. Giudice CR, D’Alessandro FJ, Galarza GA, Fernandez DS, Damia OH, Favre GA (2016) Surgical approach to vesicourethral anastomotic stricture following radical prostatectomy. Actas Urol Esp 40(2):124-130. https://doi.org/10.1016/j.acuro. 2015.08.006

16. Gomez RG, Mundy T, Dubey D, El-Kassaby AW, Firdaoessaleh, Kodama R, Santucci R (2014) SIU/ ICUD consultation on urethral strictures: pelvic fracture urethral injuries. Urology 83(3):48-58 https://doi.org/10.1016/j.urology.2013.09.023

17. Greenberg SH (1979) Male reproductive tract sequelae of gonococcal and nongonococcal urethritis. Arch Androl 3(4):317-319. https://doi. org/10.3109/01485017908988422

18. Horuz R, Goktas C, Cetinel AC, Akca O, Selimoglu A, Albayrak S (2013) The use of suprapubic cystoscopy in perineal urethroplasty. Turk J Urol 39(4):244-248. https://doi.org/10.5152/tud.2013. 061

19. Hosseini SJ, Kaviani A, Jabbari M, Hosseini MM, Haji-Mohammadmehdi-ArbabA, Simaei NR (2006) Diagnostic application of flexible cystoscope in pelvic fracture urethral distraction defects. Urol J 3(4):204-207

20. Hussain M, Greenwell TJ, Shah J, Mundy A (2004) Long-term results of a self-expanding wallstent in the treatment of urethral stricture. BJU Int 94(7):1037-1039. https://doi.org/10.1111/j.1464410X.2004.05100.x

21. Konnak JW, Kogan BA (1980) Otis internal urethrotomy in the treatment of urethral stricture disease. J Urol 124(3):356-358. https://doi.org/10. 1016/s0022-5347(17)55445-2

22. Kowalewski KF, Tapking C, Hetjens S, Nickel F, Mandel P, Ritter M, Kriegmair MC (2017) Interrupted versus continuous suturing for vesicourethral anastomosis during radical prostatectomy: protocol for a systematic review and meta-analysis. BMJ Open 7(11):e19823. https://doi.org/10.1136/ bmjopen-2017-019823

23. Kropfl D, Tucak A, Prlic D, Verweyen A (1998) Using buccal mucosa for urethral reconstruction in primary and re-operative surgery. Eur Urol 34(3):216-220. https://doi.org/10.1159/ 000019716

24. Latini JM, McAninch JW, Brandes SB, Chung JY, Rosenstein D (2014) SIU/ICUD consultation on urethral strictures: epidemiology, etiology, anatomy, and nomenclature of urethral stenoses, strictures, and pelvic fracture urethral disruption injuries. Urology 83(3):S1-7. https://doi.org/10. 1016/j.urology.2013.09.009

25. Levy A, Browne B, Fredrick A, Stensland K, Bennett J, Sullivan T, Rieger-Christ KM, Vanni AJ (2019) Insights into the pathophysiology of urethral stricture disease due to lichen sclerosus: comparison of pathological markers in lichen sclerosus induced strictures vs nonlichen sclerosus induced strictures. J Urol 201(6):1158-1163. https://doi.org/10.1097/JU.0000000000000155

26. Liu JS, Walker K, Stein D, Prabhu S, Hofer MD, Han J, Yang XJ, Gonzalez CM (2014) Lichen sclerosus and isolated bulbar urethral stricture disease. J Urol 192(3):775-779. https://doi.org/10.1016/j.juro. 2014.03.090

27. Lubahn JD, Zhao LC, Scott JF, Hudak SJ, Chee J, Terlecki R, Breyer B, Morey AF (2014) Poor quality of life in patients with urethral stricture treated with intermittent self-dilation. J Urol 191(1):143-147. https://doi.org/10.1016/j.juro.2013.06.054

28. Lumen N, Hoebeke P, Willemsen P, De Troyer B, Pieters R, Oosterlinck W (2009) Etiology of urethral stricture disease in the 21 st century. J Urol 182(3):983-987. https://doi.org/10.1016/j.juro. 2009.05.023

29. Maciejewski C, Rourke K (2015) Imaging of urethral stricture disease. Transl Androl Urol 4(1):2-9. https://doi.org/10.3978/j.issn.2223-4683.2015. 02.03

30. Mandhani $A$, Chaudhury $H$, Kapoor R, Srivastava A, Dubey D, Kumar A (2005) Can outcome of internal urethrotomy for short segment bulbar urethral stricture be predicted? J Urol 173(5):1595-1597. https://doi.org/10.1097/01.ju. 0000154347.24230.f1

31. Manzoni G, Bracka A, Palminteri E, Marrocco G (2004) Hypospadias surgery: when, what and by whom? BJU Int 94(8):1188-1195. https://doi.org/ 10.1046/j.1464-410x.2004.05128.x

32. McCallum RW, Colapinto V (1979) The role of urethrography in urethral disease. Part I. Accurate radiological localization of the membranous urethra and distal sphincters in normal male subjects. J Urol 122(5):607-611. https://doi.org/ 10.1016/s0022-5347(17)56525-8

33. McMillan A, Pakianathan $\mathrm{M}, \mathrm{Mao} \mathrm{JH}$, Macintyre CC (1994) Urethral stricture and urethritis in men in Scotland. Genitourin Med 70(6):403-405. https:// doi.org/10.1136/sti.70.6.403

34. Meeks JJ, Barbagli G, Mehdiratta N, Granieri MA Gonzalez CM (2012) Distal urethroplasty for isolated fossa navicularis and meatal strictures. BJU Int 109(4):616-619. https://doi.org/10.1111/j. 1464-410X.2011.10248x

35. Mundy AR, Andrich DE (2011) Urethral strictures. BJU Int 107(1):6-26. https://doi.org/10.1111/j. 1464-410X.2010.09800.X

36. Nathan A, Mazzon G, Pavan N, De Groote R, Sridhar A, Nathan S (2019) Management of intractable bladder neck strictures following radical prostatectomy using the Memokath ${ }^{\circledR} 045$ stent. J Robot Surg. https://doi.org/10.1007/ s11701-019-01035-9

37. Pansadoro V, Emiliozzi P (1998) Internal urethrotomy. Urologe A 37(1):21-24. https://doi.org/10 1007/s001200050143

38. Pfalzgraf D, Siegel FP, Kriegmair MC, Wagener N (2017) Bladder neck contracture after radical prostatectomy: what is the reality of care? J Endourol 31(1):50-56. https://doi.org/10.1089/ end.2016.0509

39. Pfistermuller KL, McArdle AJ, Cuckow PM (2015) Meta-analysis of complication rates of the tubularized incised plate (TIP) repair. J Pediatr Urol 11(2):54-59. https://doi.org/10.1016/j.jpurol. 2014.12.006

40. Schuettfort VM, Dahlem R, Kluth L, Pfalzgraf D, Rosenbaum C, Ludwig T, Fisch M, Reiss CP (2017) Transperineal reanastomosis for treatment of highly recurrent anastomotic strictures after radical retropubic prostatectomy: extended follow-up. World J Urol 35(12):1885-1890. https:// doi.org/10.1007/s00345-017-2067-8

41. Stephenson AJ, Scardino PT, Bianco FJ Jr. DiBlasio CJ, Fearn PA, Eastham JA (2004) Morbidity and functional outcomes of salvage radical prostatectomy for locally recurrent prostate cancer after radiation therapy. J Urol 172(6 Pt 1):2239-2243. https://doi.org/10.1097/01.ju. 0000140960.63108 .39

42. Surya BV, Provet J, Johanson KE, Brown J (1990) Anastomotic strictures following radical prostatectomy: risk factors and management. J Urol 143(4):755-758. https://doi.org/10.1016/ s0022-5347(17)40082-6

43. Tam CA, Voelzke BB, Elliott SP, Myers JB, McClung CD, Vanni AJ, Breyer BN, Erickson BA, Trauma and Urologic Reconstruction Network of Surgeons (TURNS) (2016) Critical analysis of the use of uroflowmetry for urethral stricture disease surveillance. Urology 91:197-202. https://doi.org/ 10.1016/j.urology.2015.12.070

44. Veeratterapillay R, Pickard RS (2012) Longterm effect of urethral dilatation and internal urethrotomy for urethral strictures. Curr Opin Urol 22(6):467-473. https://doi.org/10.1097/MOU 0b013e32835621a2

45. Viers BR, Pagliara TJ, Shakir NA, Rew CA, FolgosaCooley L, Scott JM, Morey AF (2018) Delayed reconstruction of bulbar urethral strictures is associated with multiple interventions, longer strictures and more complex repairs. J Urol 199(2):515-521. https://doi.org/10.1016/j.juro. 2017.08.081

46. Wang R, Wood DP Jr., Hollenbeck BK, Li AY, He C, Montie JE, Latini JM (2012) Risk factors and quality of life for post-prostatectomy vesicourethral anastomotic stenoses. Urology 79(2):449-457. https://doi.org/10.1016/j.urology.2011.07.1383

47. Wessells $\mathrm{H}$, Angermeier KW, Elliott $\mathrm{S}$, Gonzalez CM, Kodama R, Peterson AC, Reston J, Rourke K, Stoffel JT, Vanni AJ, Voelzke BB, Zhao L, Santucci RA (2017) Male urethral stricture: American urological association guideline. J Urol 197(1):182-190. https://doi.org/10.1016/j.juro.2016.07.087

48. Zeller S, Vetterlein MW, Fisch M, Kluth LA (2018) Meatusenge und glanduläre Harnröhrenstriktur. Urologe 57(1):11-16. https://doi.org/10.1007/ s00120-017-0555-5

Hinweis des Verlags. Der Verlag bleibt in Hinblick auf geografische Zuordnungen und Gebietsbezeichnungen in veröffentlichten Karten und Institutsadressen neutral. 